\title{
Anisotropy Effects in Atomic-Scale Friction
}

\author{
Enrico Gnecco • Oscar Y. Fajardo · Carlos M. Pina • Juan J. Mazo
}

\begin{abstract}
The static and kinetic friction experienced by a point mass elastically driven at different angles on surface lattices with square, hexagonal, and honeycomb symme-tries are estimated by analytical and numeric calculations based on the Prandtl-Tomlinson (PT) model. Assuming a strong surface coupling, the anisotropy of static friction increases from 3.7 up to $46.3 \%$ when the density of packing of the surface atoms is reduced, but this is not the case for kinetic friction, the anisotropy of which is maxi-mal on a square lattice. Although these results have not been supported by accurate experimental verifications so far, the PT model was successfully applied to interpret anisotropy effects in the friction force profiles measured, among other surfaces, on rectangular lattices with complex unit cells and on stepped crystal surfaces.
\end{abstract}

\section{Introduction}

The influence of the sliding direction on friction has been the subject of several AFM investigations [18]. In spite of that, only a few of these studies focused on systematic measurements of the variation of the friction force with the scan angle $\varphi$. The kinetic friction $F_{\text {kin }}$ was found to peak up along well-defined crystallographic directions on pyro-litic graphite [4], on a metallic quasicrystal [5], and on a pentacene single crystal [6]. The results on graphite and the quasicrystal were reproduced with theoretical calculations based on the Prandtl-Tomlinson (PT) model [9, 10]. However, in the case of graphite, a specific assumption was necessary: the tip was supposed to be covered by several atoms rigidly joined in the same arrangement of the surface lattice, reproducing a graphene flake previously transferred from the substrate, and a good agreement with the exper-imental data could only be obtained when the number of these atoms was $N \sim 80-100$. The question arises of what happens when $N=1$, i.e., when only one atom of the tip apex is in contact with the surface. Can we determine precise expressions for the dependence of friction on the sliding direction? The answer is partially positive, as shown in Sect. 2, where an analytical formula is provided for the static friction force $F_{\text {stat }}$ experienced by an atomi-cally sharp tip sliding on a hexagonal lattice. As a result, $F_{\text {stat }}(\varphi)$ does not vary significantly with $\varphi$, and the same can be said for the kinetic friction $F_{\text {kin }}$, the angular dependence of which is calculated numerically. This result differs significantly from the strong anisotropy predicted for a large contact area. Analytical expressions can also be found for both static and kinetic friction in the paradig-matic case of a square lattice. We will also briefly discuss surface lattices with complex unit cells and the out-of-plane friction anisotropy observed when a sharp AFM tip

Keywords Nanotribology $\cdot$ Friction mechanisms $\cdot$ Stick-slip $\cdot$ Static friction $\cdot$ AFM

E. Gnecco $(\bowtie)$

Instituto Madrileño de Estudios Avanzados, IMDEA

Nanociencia, 28049 Madrid, Spain

e-mail: enrico.gnecco@imdea.org

\section{M. Pina}

Departamento de Cristalografía y Mineralogı́a, Universidad

Complutense de Madrid, 28040 Madrid, Spain

e-mail: cmpina@geo.ucm.es
O. Y. Fajardo · J. J. Mazo

Departamento de Física de la Materia Condensada and Instituto de Ciencia de Materiales de Aragón, CSIC-Universidad de Zaragoza, 50009 Zaragoza, Spain e-mail: yofar008@gmail.com

$$
\begin{aligned}
& \text { J. J. Mazo } \\
& \text { e-mail: juanjo@unizar.es }
\end{aligned}
$$


crosses a step edge. In those cases, a direct comparison with recent experimental results is possible, as shown in Sect. 3.

\section{The PT Model in 2D}

A thorough discussion of the PT model in one dimension, including historical perspectives, was recently provided by Müser [11]. Effects of surface disorders, surface defects, and temperature were studied, among others, in [12, 13]. The PT model was also repeatedly used in two dimensions (2D), where it could reproduce several friction maps acquired on surfaces of intermediate complexity such as $\mathrm{NbSe}_{2}$ [14], $\mathrm{MoS}_{2}$ [15], $\beta-\mathrm{MoTe}_{2}(001)$ [16], and graphene [17]. The PT model simply focuses on the motion of a point mass $m$ driven by a lateral spring over an empiric potential $U_{\text {int }}(x, y)$ with the periodicity of a surface crystal lattice. If the corrugation of the potential $U_{\text {int }}$ is large enough, the point mass (which may represent the tip apex of an AFM) responds to the pulling force exerted by the spring (the AFM cantilever under torsion) by an alternation of 'stick' and 'slip' phases. Here, we would like to determine the dependence $F=F(\varphi)$ of the elastic force $F$ on the scan angle $\varphi$ both at the onset of the first slip, which we define as the static friction $F_{\text {stat }}$, and averaged over long sliding distances, which can be identified with the kinetic friction $F_{\text {kin }}$.

To estimate the force $F_{\text {stat }}$, it is sufficient to calculate the equilibrium positions of the point mass as a function of time, or, equivalently, of the coordinates of the support pulling the spring across the surface. These positions are given by $\nabla U=0$, where $U(x, y ; X, Y)$ is the sum of the tip-surface interaction potential $U_{\text {int }}(x, y)$ and of the elastic potential $U_{\mathrm{el}}=(1 / 2) k(\mathbf{r}-\mathbf{R})^{2}$, with $k$ being the spring stiffness, $\mathbf{r} \equiv(x, y)$ the tip position on the surface, and $\mathbf{R} \equiv$ $(X, Y)$ the support position. The last one varies as $\mathbf{R}=\mathbf{V} t$ with time $t$, if the support is moved at a constant velocity V. The value $F_{\text {stat }}$ is reached when the equilibrium of the tip becomes unstable, i.e., when at least one of the eigenvalues of the Hessian matrix $\partial^{2} U / \partial x_{i} \partial x_{j}$ becomes negative. In principle, explicit or implicit analytic expressions for $F_{\text {stat }}(\varphi)$ can be found for any shape of the interaction potential $U_{\text {int }}$.

To estimate the force $F_{\text {kin }}$ one has to solve the equation of motion of the point mass. In such a case, a damping term $F_{\text {damp }}=-\gamma \dot{\mathbf{r}}$ must be introduced to describe the coupling with phonons and electrons in the substrate [11]:

$m \ddot{\mathbf{r}}+\gamma \dot{\mathbf{r}}+k(\mathbf{r}-\mathbf{R})=-\nabla U_{\mathrm{int}}(\mathbf{r} ; t)$.

Depending on the values of the damping coefficient $\gamma$, different tip trajectories are possible, and analytic solutions are difficult to determine. An exception is given by a lattice with square symmetry, which is the first case that we discuss.

\subsection{Square Lattices}

The simplest potential with square symmetry is obtained by overlapping two plane waves with periodicity $a$ and rotated by $90^{\circ}$ with respect to each other (Fig. 1a):

$U_{\mathrm{int}}=-U_{0}(\cos x+\cos y)$

(for the sake of simplicity, we 'measure' every length in units of $2 \pi / a$, where $a$ is the lattice constant). Using the procedure described above it turns out that the first instability (when $\varphi<45^{\circ}$ ) is reached at the support position $X_{\mathrm{c}}=\sqrt{\eta^{2}-1}+\arccos \left(-\frac{1}{\eta}\right)$,

where $\eta=(2 \pi)^{2} U_{0} / k a^{2}$. Note that the stick-slip regime is possible only if $\eta>1$. If $\eta<1$ the tip follows the support with alternate phases of acceleration and deceleration but without abrupt jumps. When $\varphi<45^{\circ}, x_{\mathrm{c}}=\arccos (-1 / \eta)$ is the $x$ coordinate of the tip at the critical position.

Here, we only consider the case of a strong tip-surface interaction, when $\eta \gg 1$. In such a case $X_{\mathrm{c}} \simeq U_{0} / k$ and, from the equilibrium condition $\nabla U(x, y)=0$ for a given value $(X, Y)$ of the support position, it turns out that the tip trajectory before the critical point is approximately given by
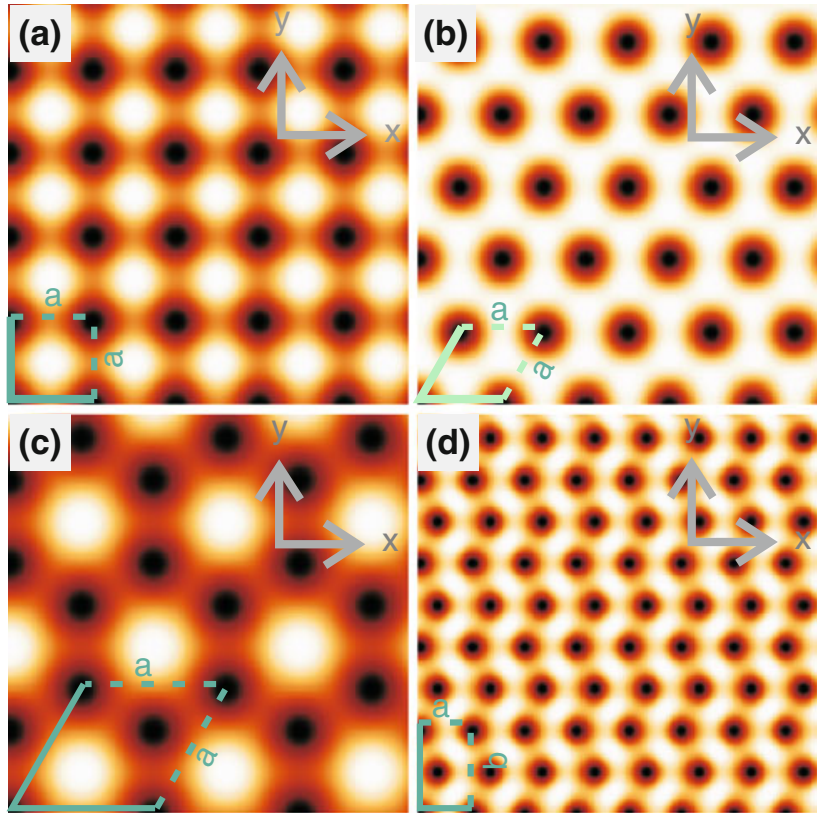

Fig. 1 Surface potentials associated to the lattices a with square symmetry, b with hexagonal symmetry, $\mathbf{c}$ with honeycomb symmetry, and $\mathbf{d}$ with a rectangular unit cell containing two atoms, respectively described by the Eqs. 2, 6, 10, and 11 in the text 
$\frac{\sin y}{\sin x}=\tan \varphi$.

The elastic force is $\mathbf{F} \equiv-\nabla U_{\mathrm{el}}=\nabla U_{\text {int }}$ or, in components,

$F_{x}=\frac{2 \pi U_{0}}{a} \sin x, \quad F_{y}=\frac{2 \pi U_{0}}{a} \sin y$

(these relations being valid for any value of $\eta$ ). The static friction can be expressed as a function of the scan direction using (3):

$F_{\text {stat }}=\left.\sqrt{F_{x}^{2}+F_{y}^{2}}\right|_{x=x_{\mathrm{c}}}=\frac{2 \pi U_{0}}{a} \times \begin{cases}\frac{1}{\cos \varphi} & \left(0^{\circ}<\varphi<45^{\circ}\right) \\ \frac{1}{\sin \varphi} & \left(45^{\circ}<\varphi<90^{\circ}\right)\end{cases}$

and similarly when $\varphi<0^{\circ}$ or $\varphi>90^{\circ}$ (see Fig. 2). The force (4) has a minimum when $\varphi=0^{\circ}$ and a maximum when $\varphi=45^{\circ}$. The maximum shows up as a cusp. More general calculations, without the limitation $\eta \gg 1$, were presented in Ref. [18]. If $\eta \rightarrow 1$ the static friction force was found to vary with the initial position of the point mass. When plotted as a function of the scan angle $\varphi$ the possible values of $F_{\text {stat }}$ form a broad band within two limit curves, which collapse onto the single curve (4) when $\eta \gg 1$.

When determining the kinetic friction force, we note that, due to the absence of cross terms in the potential (2), the average values of the $x$ and $y$ components of the elastic force are the same: $\bar{F}_{x}=\bar{F}_{y}$ (if $\varphi \neq 0^{\circ}$ nor $90^{\circ}$ ). When $\eta \gg 1$, these values are equal to $2 \pi U_{0} / a$. Since the kinetic friction is given by the projection of the average elastic force $\overline{\mathbf{F}}$ along the scan direction, one easily finds that

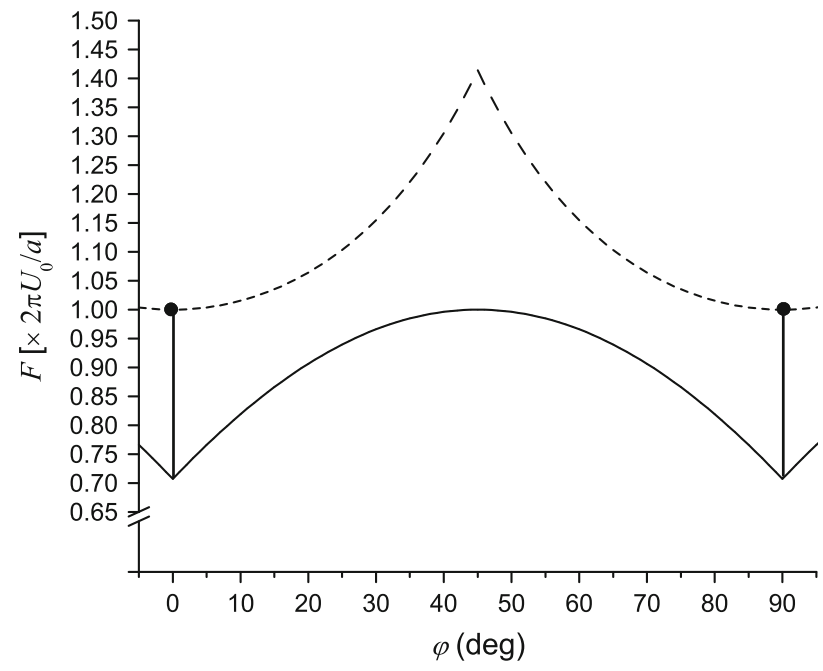

Fig. 2 Angular dependence of the static (dashed curve) and kinetic friction (continuous curve) corresponding to the square surface potential in Fig. 1a and evaluated according to the Eqs. 4 and 5
$F_{\text {kin }}=\frac{2 \pi U_{0}}{a} \cos \left(45^{\circ}-\varphi\right)$.

The force (5) has also a minimum when $\varphi \rightarrow 0^{\circ}$ or $\varphi \rightarrow$ $90^{\circ}$ and a maximum when $\varphi=45^{\circ}$, but in this case, the cusp corresponds to the minimum value and not to the maximum. In the special cases $\varphi=0^{\circ}$ or $90^{\circ}$ the tip moves along a straight line. When $\eta \gg 1$ the value of $F_{\text {kin }}(0)$ tends to $F_{\text {stat }}(0) \simeq 2 \pi U_{0} / a$, and shows up as a singularity. The result (5) is confirmed by numeric solutions of the equation of motion (1) for a broad range of values of the damping coefficient $\gamma$ [18]. When plotting the tip trajectories estimated from these calculations it can be seen that, when a critical position is reached, the point mass suddenly jumps along a straight line oriented at $0^{\circ}$ (if $\varphi<45^{\circ}$ ) or $90^{\circ}$ (if $\varphi>45^{\circ}$ ). For both static and kinetic friction, the ratio between the extremal values is $F_{\max } / F_{\min }=\sqrt{2}$ and a considerable anisotropy is thus expected.

\subsection{Hexagonal Lattices}

The simplest surface lattice with hexagonal periodicity is defined by overlapping three plane waves, rotated by $60^{\circ}$ (Fig. 1b):

$U_{\text {int }}=-\frac{U_{0}}{2}\left[\cos \left(x-\frac{y}{\sqrt{3}}\right)+\cos \left(x+\frac{y}{\sqrt{3}}\right)+\cos \left(\frac{2 y}{\sqrt{3}}\right)\right]$

The same procedure adopted for the square lattice, leads to precise expressions for the angular dependence of the static friction also in this case [19]. In particular, when $\eta \gg 1$ :

$F_{\text {stat }}=\frac{2 \pi U_{0}}{\sqrt{3} a} \sqrt{c_{x}^{2}+4 c_{x} c_{y}+7 c_{y}^{2}-4 c_{x}^{2} c_{y}^{2}-4 c_{x} c_{y}^{3}-4 c_{y}^{4}}$,

where the quantities $c_{x} \equiv \cos x$ and $c_{y} \equiv \cos (y / \sqrt{3})$, at the critical position, are related by

$c_{x}=c_{y}-2 c_{y}^{3} \pm \sqrt{1-4 c_{y}^{4}+4 c_{y}^{6}}$.

The tip trajectory, when $\eta \rightarrow \infty$, is given by

$\tan \varphi=\frac{s_{y}\left(c_{x}+2 c_{y}\right)}{s_{x} c_{y}}$

with $s_{x} \equiv \sin x$ and $s_{y} \equiv \sin (y / \sqrt{3})$, and an explicit but not trivial representation of $F_{\text {stat }}$ versus $\varphi$ is again possible. From the expressions (7-9), calculated for $\varphi=0^{\circ}$ and $\varphi=$ $30^{\circ}$, the ratio between the maximum and the minimum values of the static friction turns out to be 1.037. Small variations of $F_{\text {stat }}(\varphi)$ are estimated also for lower values of $\eta(>2)$ [19]. Thus, oppositely to the case of a square lattice, 
the static friction force on the hexagonal potential (6) is almost independent of the scan direction.

The behavior of the kinetic friction $F_{\text {kin }}$ has been calculated as a function of $\varphi$ by solving the equation of motion (1) when $\eta=100$ : see Fig. 3. Here, two singular points appear. The first one, at $\varphi=0^{\circ}$, has the same origin of the singularity observed on the square lattice. If the tip moved quasi-statically, this value would be equal to $F_{\text {stat }}(0)=2 \pi U_{0} / a$. The second singularity is due to the straight pathway of the tip when $\varphi=30^{\circ}$ (and $x=y \sqrt{3}$ ). However, a section of the potential along this direction is not sinusoidal. Two additional wells appear, and we cannot say that $F_{\text {kin }}\left(30^{\circ}\right)$ tends to $F_{\text {stat }}\left(30^{\circ}\right)$. Indeed, the isolated point at $\varphi=30^{\circ}$ is lower than $F_{\text {kin }}\left(\varphi \rightarrow 30^{\circ}\right)$. Excluding the singularities, $F_{\text {kin }}$ increases from $\varphi=0^{\circ}$ to $\varphi=30^{\circ}$ and the ratio $F_{\text {max }} / F_{\text {min }} \simeq 1.09$ is larger than the corresponding ratio for the static friction but still well below the corresponding ratio obtained on the square lattice. For comparison, Verhoeven et al. reported larger variations of the kinetic friction (in the order of 20-30\%) with a 96-atom flake attached to the tip apex [9].

\subsection{Lattices with Complex Unit Cells}

In view of a comparison with experimental results, we briefly discuss also two surface lattices with complex unit cells: (i) A honeycomb lattice obtained from a hexagonal lattice with two atoms per unit cell and (ii) A rectangular lattice with two atoms per unit cell. These cases may resemble the frictional response of graphite and of specific cleavage surfaces of minerals such as calcite and dolomite.

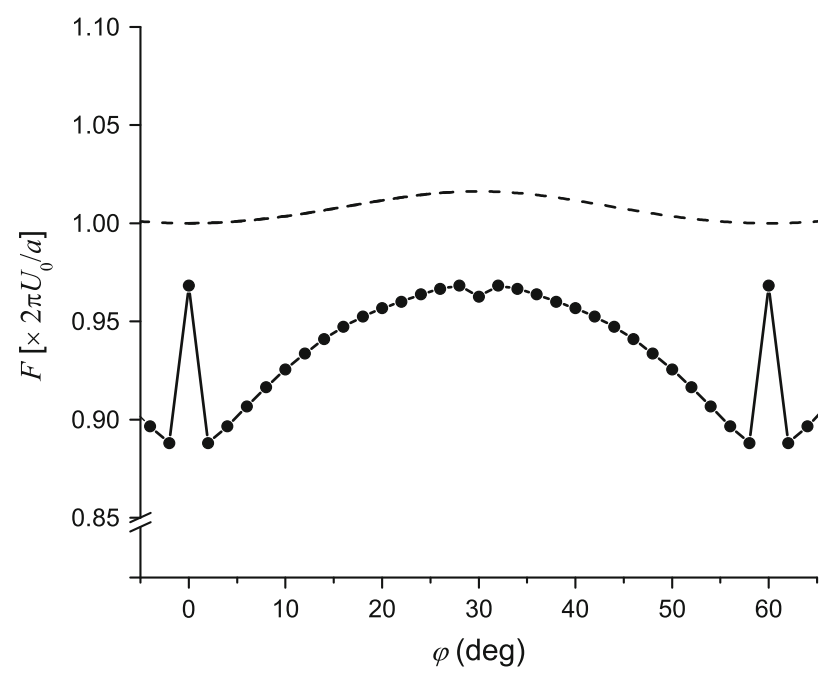

Fig. 3 Angular dependence of the static friction (upper curve) corresponding to the hexagonal surface potential in Fig. $1 \mathrm{~b}$ and evaluated with the formulas (7-9). The dots connected by the lower curve correspond to the kinetic friction estimated from numeric solutions of the equation of motion of the tip

\subsubsection{Honeycomb Lattices}

For the honeycomb lattice, we use the representation introduced by Braun and Ferrando [20] (Fig. 1c):

$$
\begin{aligned}
U_{\mathrm{int}}= & 0.80 U_{0}\left(\frac{3}{2}-\cos \frac{x}{\sqrt{3}} \cos \frac{y}{3}-\frac{1}{2} \cos \frac{2 y}{3}\right) \\
& \times\left(\frac{3}{2}+\cos \frac{x}{\sqrt{3}} \cos \frac{y+\pi}{3}+\frac{1}{2} \sin \frac{4 y+\pi}{6}\right) .
\end{aligned}
$$

Here, analytical estimations of $F_{\text {stat }}$ are quite difficult, except in the two directions $\varphi=30^{\circ}$ and $\varphi=-30^{\circ}$, where $F_{\text {stat }}$, as clear from Fig. $1 \mathrm{c}$, is expected to reach its minimum and maximum values (or vice versa, depending on which of the two atoms in the unit cell is taken as starting point). The anisotropy of static friction turns out to be $46.3 \%$ when $\eta \gg 1$ (and even larger, up to $\sim 75 \%$ when $\eta=2.5$, as estimated in [19]). Note that $F_{\text {stat }}$ has a periodicity of $120^{\circ}$. The situation is quite different for kinetic friction. Here, systematic calculations of $F_{\text {kin }}$ versus $\varphi$ lead to a lower anisotropy (Fig. 4). Excluding the singularities at $\varphi=0^{\circ}$ and $\varphi=30^{\circ}$, the ratio $F_{\max } / F_{\min } \simeq 1.15$. Furthermore, the kinetic friction has a periodicity of $60^{\circ}$. Combining with the results in Sects. 2.1 and 2.2, we may thus conclude that the anisotropy of static friction increases when the atomic packing density decreases, although this is not necessarily the case for kinetic friction.

\subsubsection{Rectangular Lattices}

A convenient representation of the interaction experienced by a sharp tip sliding on a rectangular lattice with

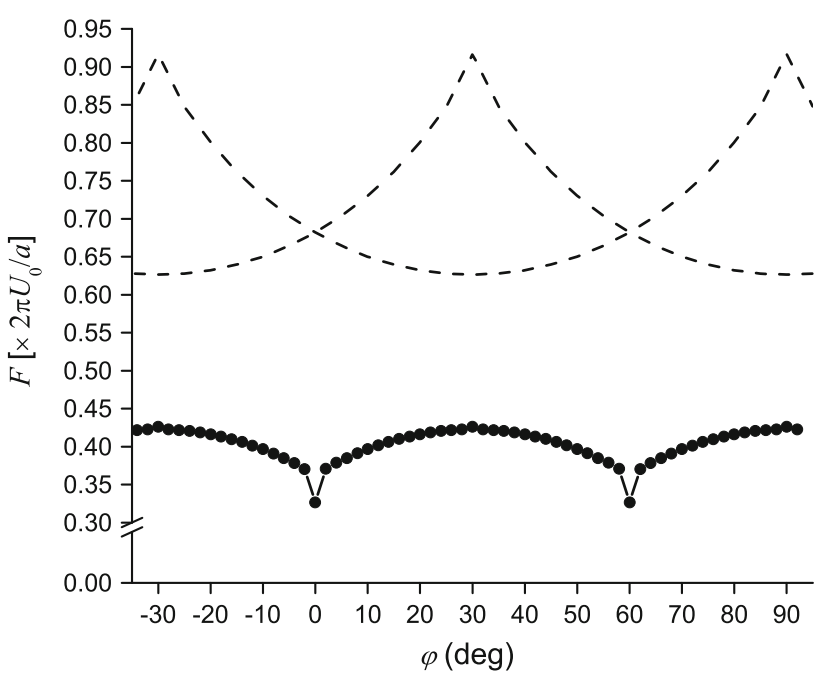

Fig. 4 Angular dependence of the static friction (dashed curves) and kinetic friction (lower curve) corresponding to the honeycomb surface potential in Fig. 1c and evaluated numerically. Depending on the starting location in the unit cell, static friction is described by two different curves 
$M$ pinning centers per cell is given by a series of potential wells with Gaussian shape (Fig. 1d):

$U_{\text {int }}=-\sum_{i=1}^{M} U_{0, i} \exp \left[-\left(\frac{\sin ^{2} \beta\left(x-x_{i}\right)}{\beta^{2} r^{2}}+\frac{\sin ^{2}\left(y-y_{i}\right)}{r^{2}}\right)\right]$,

where $\beta$ is the ratio between the lattice constants of the rectangular cell and $r$ defines the width of the wells. When $M=2, \beta \simeq 1.6$ and $\left(x_{2}-x_{1}, y_{2}-y_{1}\right)=(3 \pi / 4, \pi / 2)$ this potential describes quite well the scenario observed when a sharp AFM tip slides on the (104) cleavage faces of calcite and dolomite crystals and sticks to the oxygen atoms protruding out of the surfaces [21]. Here, we haven't performed a systematic analysis of the angle dependence of the frictional forces. Nevertheless, an interesting result is found, as detailed in Ref. [21]. Although the kinetic friction is the same when scanning at an angle $\varphi=0^{\circ}$ or $\varphi=90^{\circ}$ with respect to the $x$ axis, the friction profile (i.e., the friction force as a function of time) looks quite different in the two directions. Provided that the interaction is, once again, strong enough, two friction peaks per unit cell are observed when $\varphi=90^{\circ}$, and only one peak when $\varphi=0^{\circ}$, which is the same as observed experimentally. In other words, anisotropy shows up in the number of sites where the tip becomes coupled to the surface.

\subsection{Out-of-Plane Anisotropy}

A different kind of anisotropy is observed when an AFM tip climbs up or descends along an obstacle, for instance a monatomic step edge on a crystal surface. The friction profile is not necessarily symmetric when the scan direction is inverted. In fact, kinetic friction can either (i) increase in both directions [22] or (ii) increase upwards but decrease downwards [23]. This situation, which may be called out-of-plane friction anisotropy, can also be studied with the PT model. The step edge represents a rupture in the symmetry of the surface lattice. The symmetry rupture results in both a so-called Ehrlich-Schwöbel barrier [24, 25 ] and in an asymmetric potential well. In a phenomenological approach, one can replace the barrier with a Gaussian profile $U_{\text {barrier }}$ and represent the asymmetric well with the function

$U_{\text {well }}=U_{1}\left[-\operatorname{erf}\left(\frac{x}{b_{1}}\right)+\operatorname{erf}\left(\frac{x}{b_{2}}\right)\right]$,

where the parameters $b_{1}$ and $b_{2}\left(>b_{1}\right)$ are slightly larger than the lattice constant. Depending on the ratio between the amplitudes $U_{\text {barrier }}$ and $U_{\text {well }}$, either the case (i) is observed (when $U_{\text {barrier }}>U_{\text {well }}$ ) or the case (ii) (when $\left.U_{\text {barrier }}<U_{\text {well }}\right)$. This result has been recently confirmed by friction measurements across monatomic step edges of a
$\mathrm{NaCl}(001)$ surface [23]. The case (i) was indeed observed using an atomically sharp tip and a normal load close to the jump-off value. Otherwise, when the tip is blunt and the surface coupling becomes stronger, also at low loads, the case (ii) is observed.

\section{Discussion}

Altogether, the previous results show one fact: The PT model can predict a series of interesting effects, from a strong anisotropy of friction on square lattices (compared to hexagonal lattices) to a variable number of 'friction peaks' depending on the scan direction. Whether these predictions correspond or not to the reality can only be tested by carefully planned AFM measurements. Clean environmental conditions, such as ultra-high vacuum (UHV), are required to measure the variations of the static and kinetic friction with the scan angle $\varphi$. Furthermore, the tip apex should not change when the angle $\varphi$ is varied. Unfortunately, these conditions are rarely achieved in practice. In order to vary the scan angle $\varphi$, one has to rotate either the surface sample or the cantilever, but none of the AFM setups in UHV at our knowledge can do that without breaking the vacuum. Alternatively, one can keep the orientation between cantilever and surface, and change the sliding direction. However, even the simplest cantilever geometry (the rectangular shape) introduces problems such as cantilever buckling. This problem has been thoroughly discussed in a recent paper by Campione et al. [26], where the apparent height variations due to buckling were related to the friction component along the cantilever axis and used to determine the so-called friction hodograph on anisotropic surfaces.

We should also notice that our calculations were implicitly performed at zero temperature, and thermal effects may significantly modify many conclusions, most noticeably the singularities observed along the main crystallographic directions. As a first attempt to study these changes, we have solved the equation of motion of the nanotip introducing a noise term on the R.H.S. of Eq. 1 satisfying the fluctuation-dissipation theorem. The average value of the kinetic friction is reduced overall at a finite temperature $T=293 \mathrm{~K}$, as expected [27]. Furthermore, the simulated trajectories in Fig. 5a show that the tip alternatively jumps at $0^{\circ}$ and $90^{\circ}$ when it is driven with an angle of $45^{\circ}$ on the square lattice. Similarly, it jumps at $0^{\circ}$ and $60^{\circ}$ when the scan direction is oriented at $30^{\circ}$ on the hexagonal lattice (Fig. 5b). The corresponding singularity in Fig. 3 is also removed. This is not the case for the peaks at $0^{\circ}$ for both the square and hexagonal lattices, which correspond to a stable path of the tip apex. Here, the only difference with $T=0 \mathrm{~K}$ is the appearance of vibrations 

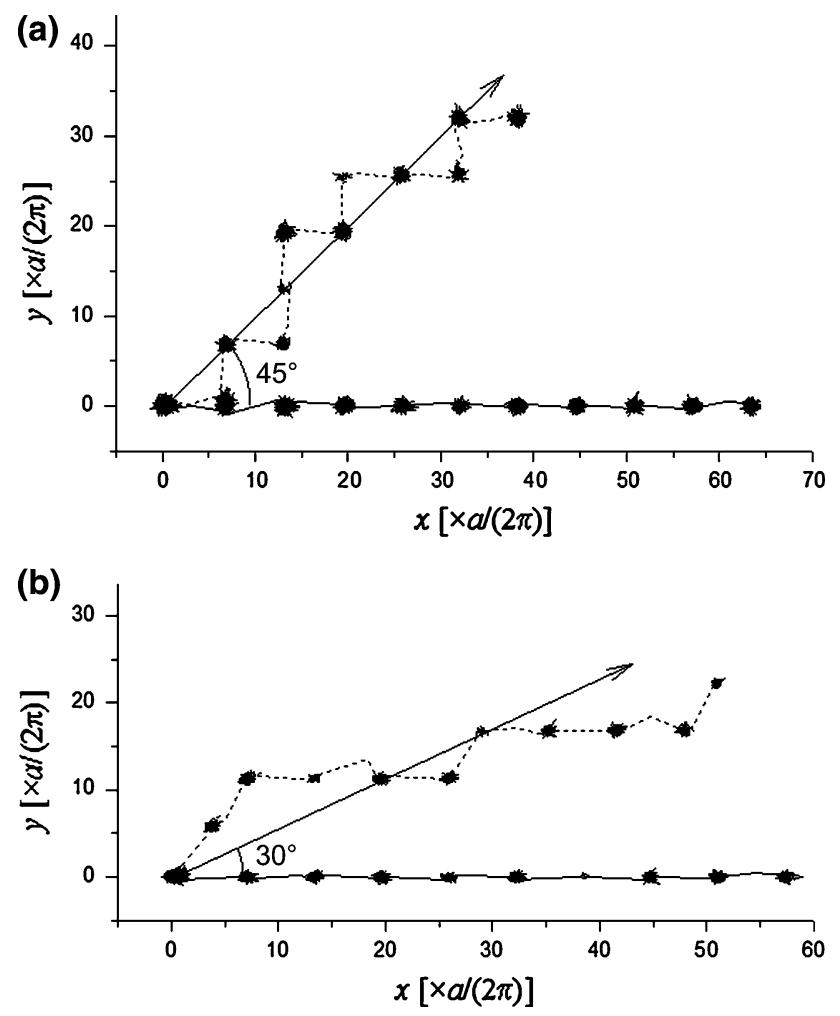

Fig. 5 Simulated tip trajectories at $T=293 \mathrm{~K}$ a on the square potential (2) and $\mathbf{b}$ on the hexagonal potential (6). The tip jumps at $0^{\circ}$ when it is pulled along the $x$ direction (continuous curves), and alternatively at $0^{\circ}$ and $90^{\circ}$ (or $60^{\circ}$ ) when it is pulled with an angle of $45^{\circ}$ (or $30^{\circ}$ ) with respect to the $x$ axis (dashed curves)

Table 1 Degree of friction anisotropy as a function of the number of first neighbors

\begin{tabular}{lll}
\hline No. of first neighbors & $\begin{array}{l}\text { Static anisotropy } \\
(\%)\end{array}$ & $\begin{array}{l}\text { Kinetic anisotropy } \\
(\%)\end{array}$ \\
\hline 6 & 3.7 & $\sim 9$ \\
4 & 41.4 & 41.4 \\
3 & 46.3 & $\sim 15$ \\
\hline
\end{tabular}

around the minima of the surface potential. A detailed analysis of thermal effects on more complex crystal structures goes beyond the purpose of this Letter and will be not discussed here.

\section{Conclusions and Outlook}

In conclusion, we have extended some recent works in which the PT model was applied to predict static and kinetic friction in 2D systems. Although most of the theoretical results are still waiting for their experimental counterparts (graphene being probably the most important system to be tested), two conclusions have been recently confirmed by AFM measurements on calcite and dolomite in liquid and on $\mathrm{NaCl}$ in ultra-high vacuum. These are the selective (i.e., direction dependent) coupling between tip and surface while sliding [21], and the anisotropy of friction across monatomic step edges [23]. These results extend the range of application of the PT model and open the path to new exciting research on this topic.

Acknowledgments Prof. Riccardo Ferrando from the University of Genova is gratefully acknowledged for scientific discussion. Our collaboration was promoted thanks to the EUROCORES programme 'Friction and Adhesion in Nanomechanical Systems' (FANAS) of the European Science Foundation. OYF and JJM acknowledge Spain MICINN under Projects No. FIS2008-01240 and FIS2011-25167 cofinanced by FEDER funds.

\section{References}

1. Overney, R.M., Takano, H., Fujihira, M., Paulus, W., Ringsdorf, H.: Anisotropy in friction and molecular stick-slip motion. Phys. Rev. Lett. 72, 3546-3549 (1994)

2. Takano, H., Fujihira, M.: Study of molecular scale friction on stearic acid crystals by friction force microscopy. J. Vac. Sci. Technol. B 14, 1272-1275 (1996)

3. Liley, M., Gourdon, D., Stamou, D., Meseth, U., Fischer, T.M., Lautz, C., Stahlberg, H., Vogel, H., Burnham, N.A., Duschl, C.: Friction anisotropy and asymmetry of a compliant monolayer induced by a small molecular tilte. Science 280, 273-275 (1998)

4. Dienwiebel, M., Verhoeven, G.S., Pradeep, N., Frenken, J.W.M., Heimber, J.A., Zandbergen, H.W.: Superlubricity of graphite. Phys. Rev. Lett. 92(1-4), 126101 (2004)

5. Park, J.Y., Ogletree, D.F., Salmeron, M., Ribeiro, R.A., Canfield, P.C., Jenks, C.J., Thiel, P.A.: High frictional anisotropy of periodic and aperiodic directions on a quasicrystal surface. Science 309, 1354-1356 (2005)

6. Kalihari, V., Haugstad, G., Frisbie, C.D.: Distinguishing elastic shear deformation from friction on the surfaces of molecular crystals. Phys. Rev. Lett. 104(1-4), 086102 (2010)

7. Campione, M., Fumagalli, E.: Friction anisotropy of the surface of organic crystals and its impact on scanning force microscopy. Phys. Rev. Lett. 105(1-4), 166103 (2010)

8. Fessler, G., Zimmermann, I., Glatzel, T., Gnecco, E., Steiner, P., Roth, R., Keene, T.D., Liu, S.X., Decurtins, S., Meyer, E.: Orientation dependent molecular friction on organic layer compound crystals. Appl. Phys. Lett. 98(1-3), 083119 (2011)

9. Verhoeven, G.S., Dienwiebel, M., Frenken, J.W.M.: Model calculations of superlubricity of graphite. Phys. Rev. B 70(1-10), 165418 (2004)

10. Filippov, A.E., Vanossi, A., Urbakh, M.: Origin of friction anisotropy on a quasicrystal surface. Phys. Rev. Lett. 104(1-4), 074302 (2010)

11. Müser, M.H.: Velocity dependence of kinetic friction in the Prandtl-Tomlinson model. Phys. Rev. B 84(1-13), 125419 (2011)

12. Fajardo, O.Y., Mazo, J.J.: Effects of surface disorder and temperature on atomic friction. Phys. Rev. B 82(1-7), 035435 (2010)

13. Fajardo, O.Y., Mazo, J.J.: Surface defects and temperature on atomic friction. J. Phys.: Condens. Matt. 23, 355008 (2011)

14. Kerssemakers, J., De Hosson, J.T.M.: Atomic-force microscopy imaging of transition-metal layered compounds-a 2-dimensional stick-slip system. Appl. Phys. Lett. 67, 347-349 (1995)

15. Hölscher, H., Schwarz, U.D., Wiesendanger, R.: Modelling of the scan process in lateral force microscopy. Surf. Sci. 375, 395-402 (1997) 
16. Hölscher, H., Raberg, W., Schwarz, U.D., Hasbach, A., Wandelt, K., Wiesendanger, R.: Imaging of sub-unit-cell structures in the contact mode of the scanning force microscope. Phys. Rev. B 59, 1661-1664 (1999)

17. Steiner, P., Gnecco, E., Filleter, T., Gosvami, N.N., Maier, S., Meyer, E., Bennewitz, R.: Atomic friction investigations on ordered superstructures. Trib. Lett. 39, 321-327 (2010)

18. Steiner, P., Roth, R., Gnecco, E., Baratoff, A., Meyer, E.: Angular dependence of static and kinetic friction on alkali halide surfaces. Phys. Rev. B 82(1-9), 205417 (2010)

19. Gnecco, E.: Quasi-isotropy of static friction on hexagonal surface lattices. Europhys. Lett. 91(1-6), 66008 (2010)

20. Braun, O.M., Ferrando, R.: Role of long jumps in surface diffusion. Phys. Rev. E 65(1-11), 061107 (2002)

21. Pina, C.M., Miranda, R., Gnecco, E.: Anisotropic surface coupling while sliding on dolomite and calcite crystals. Phys. Rev. B 85(1-4), 073402 (2012)
22. Hölscher, H., Ebeling, D., Schwarz, U.D.: Friction at atomicscale surface steps: Experiment and theory. Phys. Rev. Lett. 101(1-4), 246105 (2008)

23. Steiner, P., Gnecco, E., Krok, F., Budzioch, J., Walczak, L., Konior, J., Szymonski, M., Meyer, E.: Atomic-scale friction on stepped surfaces of ionic crystals. Phys. Rev. Lett. 106(1-4), 186104 (2011)

24. Ehrlich, G., Hudda, F.G.: Atomic view of surface self-diffusion: Tungsten on tungsten. J. Chem. Phys. 44, 1039-1049 (1966)

25. Schwöbel, R.L., Shipsey, E.J.: Step motion on crystal surfaces. J. Appl. Phys. 37, 3682-3686 (1966)

26. Campione, M., Trabattoni, S., Moret, M.: Nanoscale mapping of frictional anisotropy. Trib. Lett. 45, 219-224 (2012)

27. Zhao, X., Phillpot, S.R., Sawyer, W.G., Sinnott, S.B., Perry, S.S.: Transition from thermal to athermal friction under cryogenic conditions. Phys. Rev. Lett. 102(1-4), 186102 (2009) 